Revue européenne des sciences sociales

European Journal of Social Sciences

XXXIX-120| 2001

Autour de l'épistémologie de la sociologie et de la sociologie des sciences : des débats, un réexamen

\title{
Pour continuer le débat sur la scientificité des sciences sociales
}

Jacqueline Feldman

\section{CpenEdition}

Journals

Édition électronique

URL : http://journals.openedition.org/ress/662

DOI : $10.4000 /$ ress.662

ISSN : 1663-4446

Éditeur

Librairie Droz

Édition imprimée

Date de publication : 1 juillet 2001

Pagination : 191-222

ISBN : 2-600-00610-9

ISSN : 0048-8046

Référence électronique

Jacqueline Feldman, «Pour continuer le débat sur la scientificité des sciences sociales », Revue européenne des sciences sociales [En ligne], XXXIX-120 | 2001, mis en ligne le 15 décembre 2009, consulté le 21 décembre 2020. URL : http://journals.openedition.org/ress/662 ; DOI : https://doi.org/ $10.4000 /$ ress.662 
Jacqueline FELDMAN

\title{
POUR CONTINUER LE DÉBAT SUR LA SCIENTIFICITÉ DES SCIENCES SOCIALES
}

\author{
1. \\ DEUX FOIS DEUX AXIOMES \\ SUR LES DIFFÉRENCES ENTRE LES SCIENCES EXACTES \\ ET LES SCIENCES SOCIALES
}

\begin{abstract}
On entend par «axiomes» des propositions évidentes et premières, à partir desquelles on peut déduire d'autres propositions. Pour ce qui est du caractère d'évidence, il peut ressortir d' une décision personnelle qui est faite, et dont on espère que d'autres partageront le choix. Cette décision consiste à refuser de discuter l'axiome, afin de pouvoir partir de quelque chose, et de construire à partir de là. D'où le caractère premier, au point de vue du déroulement logique. Cependant, ce caractère «premier» est paradoxal: en effet, l'importance des axiomes est telle dans le déroulement de l'argumentation (cas faible), ou de la démonstration (cas fort, celui des mathématiques) que leur ensemble représente en quelque sorte le cour même de l' univers que l'on prétend déduire de lui. Il en serait l' "essence». Or, pour arriver à trouver l'essence d'un univers, il faut le bien connaître, l' avoir parcouru de part en part. Euclide n'a axiomatisé l'arithmétique qu'après que celle-ci ait été largement développée. Ce qui fait - d'où le côté paradoxal évoqué - que l'énoncé $d$ 'axiomes n' intervient pas vraiment en premier dans la connaissance d' un univers, mais presque en dernier, quand on est en mesure d' effectuer une reconstruction de ce qu'on connaît, de l'ordonner en partant d'éléments dits alors axiomatiques.

C'est après une longue fréquentation de la question des différences entre sciences exactes et sciences sociales que le besoin m' a prise de résumer un champ très vaste, qui a été parcouru de multiples façons par bien des auteurs prestigieux. Il m'était nécessaire d'exprimer ce qui était, pour moi, à la base de ces différences que j'éprouvais, et je n'en ai été capable qu'après avoir erré dans cet univers-là, lu, expérimenté, pensé. L'effort même de condenser ce savoir pour en arriver à quelques formules simples s'est avéré difficile, parfois carrément pénible, alors même que les résultats apparaissent bien simples, ce qui ne surprendra que ceux qui ignorent tout du travail exigeant de la simplicité.

Tels que je les obtenus, ils m'apparaissent bien comme la base qui m'était nécessaire pour examiner plus en détail et de façon mieux fondée, plus solide, les diverses différences que j'ai rencontrées lors de ces deux pratiques. Un travail qui n'est qu'ébauché ici.
\end{abstract}


On entend par sciences exactes essentiellement la physique mathématisée et les mathématiques. On pourrait y ajouter certaines parties de la biologie. De même, on pourrait en retrancher certaines parties de la physique dont la complexité est telle qu'elles se rapprocheraient plutôt du cas des sciences sociales (comme en témoignent, par exemple, les débats sur des questions aussi vitales et difficiles que le réchauffement de la planète...)

Le choix des types de science envisagés ici est fait de façon à mettre en valeur les spécificités de chacun, c' est pourquoi on s'intéresse d'abord à leurs différences les plus marquées. Il se trouve qu'il correspond à l'expérience de mon propre parcours, mais aussi à celui de la science occidentale. C'est à partir des succès de la physique mathématisée que l'on a longtemps défini la science. Encore aujourd' hui, où la biologie tend à lui ravir la primauté dans la société, elle garde un pouvoir de fascination intellectuelle pour beaucoup de praticiens des sciences sociales.

Pour arriver à faire émerger ces axiomes, il m'a fallu me libérer peu à peu des discours dominants qui m' entouraient, et où je ne me retrouvais pas, sans pouvoir pourtant les contrer tant ils étaient énoncés avec la puissance de l'évidence. C'est pourquoi il ne me parait pas inutile, dans un premier temps, d'évoquer rapidement quelques étapes de ce chemin d'élaboration.

\section{I. ÉTAPES}

\section{Deux cultures}

Tant que j'ai été physicienne, je n'ai pas eu besoin de m'intéresser à l'épistémologie. Il y avait des problèmes à résoudre, des découvertes à faire, puis à expliquer, des hypothèses à vérifier. Tout cela était suffisamment prenant pour ne pas laisser place à d'autres questions extérieures à ce travail. Certains résultats étaient clairs, admis de tous. Parfois, ils étaient spectaculaires, et ils contribuaient alors à raviver la flamme de la passion et à entretenir l'enthousiasme pour la science.

Pour tout dire, on se méfiait même de l'épistémologie, et de la philosophie. On constatait que c'étaient souvent des physiciens hors course qui s'y intéressaient, sur leurs vieux jours. La règle était de ne poser que des questions qui pouvaient être résolues, le reste étant de la «philosophie»-ou, disait-on encore avec un certain mépris, de la métaphysique.

Tout a changé quand j'ai abordé le domaine des sciences humaines et sociales. On m'y avait acceptée pour y travailler sur les méthodes mathématiques, car on avait l'espoir qu'elles allaient enfin rendre «scientifiques» ces disciplines. Mais cette approche était loin d'être évidente, et reconnue. En économie même, en dépit de sa place prépondérante, elle fait l'objet de bien des critiques, comme on sait.

Structuralisme, causalité, ces termes, à la mode alors, étaient très abstraits pour moi. Surtout, je me suis vite aperçue que mes nouveaux collègues ne se faisaient pas la même idée de la science que moi. Entre un enthousiasme qui me semblait disproportionné envers les possibilités des mathématiques, et les rejets plus ou moins idéologiques de celles-ci, il m'a fallu creuser mon propre chemin, élaborer ma propre pensée, sans la protection d'un groupe avec lequel on partage quelques positions de base communes. Mais bien vite, j'ai trouvé passionnante la question de la scientificité des sciences sociales. 
Bref, je me suis trouvée en train de faire de l'épistémologie. Ma forme d'esprit fait que je me trouve mal à l'aise dans l'abstraction des généralités philosophiques. L'épistémologie dont j'éprouvais le besoin était du type «épistémologie montante », celle que je pouvais élaborer peu à peu à partir d'une certaine mise en discours de mes pratiques: la pratique des sciences exactes, d'abord, puis l'expérience de la connaissance de la société et de l'humain, à travers la vie dans la société comme à travers les lectures savantes. Ces expériences vécues représentaient les éléments de base à partir desquels je pouvais exercer tant bien que mal la réflexion sur ce que peuvent être les divers types de savoirs.

Trois moments ont marqué l'arrivée à ce texte. Avec trois textes, le plus souvent écrits difficilement - il faut, chaque fois, extraire de la gangue quelques idées simples, qui pourraient convenir à la situation complexe où je me sentais embourbée, et soutenir d'autres avancées.

\section{Repères}

Le premier a pour titre: «Quelques repères sur les savoirs sociaux. Pour une expérience de communication».

Mon passage de l'univers des sciences physiques à celui des sciences sociales eut lieu l'automne 1967. Quelques mois plus tard, je participai avec enthousiasme au mouvement de remise en cause radicale de la société initié par les étudiants en mai 1968, et continué ensuite par le mouvement de libération des femmes. Cette remise en cause me conduisait, en particulier, à écouter les critiques faites à la science, voire, à la rationalité. Plus rien de ce que jusqu'ici j'avais pu admirer, que j'avais tenu pour acquis, n'était épargné.

Après cette entreprise de «déconstruction» systématique, il fallait reconstruire. Je passai une année à tenter de savoir où j'en étais, à effectuer ce tri entre ce qui m'avait été jusque-là proposé par la société et que j'avais reçu passivement - la valeur de la science, par exemple -, et les critiques radicales émises en ces moments de «révolution culturelle». De plus, puisque j'étais arrivée dans un domaine où la scientificité était moins que donnée et assurée, il me fallait savoir ce que je voulais faire et comment aborder mon nouveau domaine, comment m'y sentir en accord avec moi-même. Ce fut une année très difficile, je n'avais pas l'habitude de me mouvoir ainsi, seule, dans les profondeurs d'une réalité mouvante, et j'ai parfois eu l'impression de friser la folie. Mais j'ai fini par trouver des choses solides qui, aussi simples pouvaient-elles sans doute paraître, apportaient effectivement quelques solutions à ma quête.

C'est le terme «repères» que j'utilisai alors: cela signifiait de retrouver quelques éléments stables, qui ne définissaient certes pas tout un champ - ici, celui de l'épistémologie des sciences sociales, dans sa comparaison avec les sciences exactes - mais qui permettait du moins de circuler alentour sans trop courir le risque de se perdre.

Un de ces repères, c'était le choix fait de parler de «savoirs sociaux », c'est-àdire de refuser de s'en tenir uniquement aux sciences sociales instituées. Si je comparais ainsi avec la physique, il existait des savoirs portant sur le monde physique. Ces savoirs avaient été élaborés peu à peu, et on avait trouvé commode, pour aider au développement de ces connaissances, d'instituer des académies d'abord, puis divers organismes de recherche, où les «communautés scientifiques » pouvaient 
travailler, et faire le tri entre ce qui avait pu être prouvé, ce qui était faux, ce qui restait douteux. Ainsi, ce qui était «scientifique» avait acquis le sens de ce qui était vérifié par le travail en commun d'une communauté constituée, et reconnue.

Les sciences sociales ont une tout autre histoire, dans la mesure où l'institutionnalisation avait participé de leur définition. Au vu de la diversité et des désaccords existant entre les sociologues, il m'apparaissait que la sociologie était ce que produisait celui qui était reconnu en tant que sociologue. C'était ce que François Lurçat nomme une «social-science», c'est-à-dire une science qui est définie socialement avant tout. Dans l'inter-jeu entre le cognitif et le social, c'est celui-ci qui dominait ici, tandis que c'est le cognitif qui domine dans les sciences physiques, l'organisation sociale étant là pour aider le cognitif.

Parler ainsi de «savoir social», c'était affirmer une des positions sur lesquelles je ne reviendrai pas: ce qui était à la base de mon intérêt, c'était la connaissance du social, or, celle-ci pouvait exister indépendamment, et, parfois, contre ce que je nommai ici, reprenant le terme de Bourdieu, non plus «communauté » - attachée à un but commun - mais «cité scientifique», c'est-à-dire un ensemble de personnes obéissant à un certain nombre de règles communes de vie.

Il faut ensuite mentionner le style de ce texte: il se compose essentiellement de «propositions », évoquant ainsi le style des mathématiques. Il y est en effet proposé certaines assertions, que j'estime, pour ma part, vraies. Mais comme j'ai appris, par expérience, que ce qui me parait vrai n'est pas nécessairement reconnu tel par d'autres, et que je n'ai pas, ici, les moyens de la preuve, je ne pouvais qu'affirmer les «résultats » auxquels j'étais parvenue après ces mois de pratique, de réflexion et de lecture. Je ne pouvais, en guise de démonstration, que donner des «illustrations » de mes assertions, à travers les quatre champs que je connaissais bien, et sur lesquels j'appuyais ma réflexion: côté sciences exactes, les champs des mathématiques et de la physique, côté sciences sociales, les champs de la sexualité et du féminisme.

L'«expérience de communication» qui entre dans le titre, a été la suivante: j'ai soumis ces propositions à quelques-uns de mes collègues et/ou amis. Je leur demandais de définir leur position par rapport à chacune d'entre elles : étaient-ils d'accord, étaient-ils en désaccord, ou bien, ma formulation n'ayant pour eux ni sens, ni pertinence, ils n'avaient rien à en dire.

Cette expérience m'apprit que seuls, quelques-uns, en minorité, avaient pu accéder à ma demande. La plupart dérivaient sur leurs propres réflexions, plus larges, plus floues aussi. J'en déduisis que ce travail, à partir de propositions, était loin d'être considéré comme évident dans mon nouveau milieu. De fait, alors que je ne savais pas grand-chose, à ce moment, de la rhétorique, j'avais signalé la sécheresse de mon texte, son «laconisme» et, «pour tout-dire, son 'anti-rhétorisme'». Et j'avais justifié ce style, en évoquant «la vérité personnelle», que j'étais allée chercher en moi, et que je soumettais à mes lecteurs, sans autre souci de séduction que cette austérité revendiquée. Plus tard, cette expérience m'a confortée dans l'importance de la notion d' «identité épistémique », ainsi que de sa prise de conscience par chacun - dans la mesure où elle se trouverait possible. Elle partageait, dans ce cas, les lecteurs entre ceux qui acceptaient le jeu proposé et ceux qui le refusaient, ou s'y sentaient mal à l'aise.

J'évoque rapidement l'essentiel de ces propositions: le savoir est défini comme une interaction entre un «chercheur» et son «objet d'études», le tout, 
dans un «contexte donné»; cette formulation générale me semble permettre d'éviter les discussions interminables entre tenants du constructivisme, ou de l'objectivisme.

Le savoir est ancré dans un individu, je le représentais comme une bulle, pouvant contenir toutes sortes de savoirs, de niveaux différents et de qualité différente. En particulier, je distinguai les niveaux du non-savoir (un réflexe de matheux, pour qui l'élément vide, ou le zéro, doivent être intégrés dans la série, une façon de prendre en considération un ensemble vaste de situations, où les phénomènes de frontière sont importants à étudier), du senti - un savoir d'avant les mots -, des mots, qui vont servir à décrire une situation plus ou moins «donnée » - et enfin, de l'élaboration. Cette progression va dans le sens d'une connaissance toujours mieux affirmée, la connaissance dite scientifique se trouvant évidemment au niveau de l'élaboration.

\section{L'«objet»}

Le deuxième moment a consisté à arriver à cette conclusion somme toute simple, mais qui semble loin d'être perçue par tous : ce qui faisait véritablement la distinction entre les sciences exactes et les sciences sociales, c'était la nature de leurs « objets». Dans le premier cas, on a affaire à des «choses» bien définies, sur lesquelles les personnes de bonne volonté pouvaient se mettre d'accord - même si, en raison de leur spécialisation poussée, ces "personnes de bonne volonté » devaient avoir suivi un cursus particulier. Même si l'accord n'était pas toujours évident à atteindre et pouvait donner lieu à de multiples controverses, il était de l'ordre du possible, ce qui explique l'existence des controverses. Dans le second cas, les «objets » sont fluides, changeants, et il est difficile - voire, impossible de se mettre totalement d'accord. C'est cette situation qui produit par ailleurs la première règle d'or de la méthodologie des sciences sociales: «construire son objet», c'est-à-dire commencer par délimiter autant que faire se peut ce qu'il s'agit d'étudier. Le précepte est tellement intégré par les sociologues qu'ils deviennent dupes de la métaphore. Ils ne se rendent plus compte qu'il existe pour le mot «objet», un sens dit commun, et un sens figuré, et que leur «objet»n'est pas vraiment du type qui permet un consensus fort. Cette construction de l'«objet» permettrait par ailleurs au sociologue de distinguer son savoir du «savoir commun», ce qui suffirait à lui assurer la «scientificité ».

J'avais trouvé, dans un livre du physicien Marguenau, énoncée clairement cette différence: en science, les objets sont définis sans ambiguïté par les mots, avec un manque de fantaisie que refuserait le poète (citation de mémoire). Mais le philosophe, ou le sociologue auraient également leurs propres raisons de refuser cette correspondance biunivoque entre la chose et le mot: l'extrême fluidité de la réalité. La notion de «réification» a été introduite pour justement mettre en garde contre toute rigidification de la définition de la réalité. Évidemment, cette notion n'a aucune raison d'être introduite dans les sciences exactes.

C'est dans un article de 1994 que j'ai pris fermement position sur cette réalité1.

La considération de la nature spécifique des objets sociaux semble devenir une préoccupation de certains chercheurs, cf. Pierre Livet et Ruwen Ogien (dir.): L'enquête ontologique; du mode d'existence des objets sociaux. Editions de l'EHESS, Raisons pratiques, 11, 2000. 


\section{Préalables}

Le mot «science» a deux significations, une forte, et une faible. La science, c'est d'abord une certaine connaissance approfondie apportée par des spécialistes. Mais c'est aussi, dans notre société, une connaissance solide, sur laquelle on peut compter, qui a fait l'objet d'une vérification collective. Les sciences sociales jouent sur l'ambiguïté du terme due à ces deux acceptions : elles peuvent arguer du fait que leurs connaissances sont produites par une communauté s'auto-critiquant, en principe (de fait, un examen plus précis montrerait qu'on a plutôt affaire à des «groupes » allant plus ou moins ensemble dans certaines directions, et de rapports de force entre ces groupes qui combinent l'évaluation de la qualité intellectuelle d'un travail et la défense de ces directions); mais elles ne peuvent éviter la connotation forte qui accompagne le terme de «scientifique», et dont elles bénéficient chaque fois qu'elles utilisent le terme. L'appellation «sciences molles », qu'elles n'aiment pas, à juste titre, qui les distinguent des «sciences dures » - distinction qui, elle, me semble pertinente - ne suffit pas, à mes yeux, à lever cette ambiguïté, qui peut friser la malhonnêteté. Car c'est souvent sous-entendre que c'est en raison de leur jeunesse et de la difficulté particulière de leur tâche qu'elles n'arrivent «pas encore » à aboutir à ces résultats durs qui définissent la science, dans son sens fort. Le sociologue britannique Giddens compare ceux qui évoquent ce «pas encore » à des gens qui attendent, sur un quai de gare, un train qui ne viendra pas, parce qu'il n'existe pas.

Insatisfaite de ces ambiguïtés de langage, il m'était apparu que, pour parler de scientificité dans le cas des sciences sociales, certains préalables étaient nécessaires, qui n'avaient pas encore été posés tels, en raison de la hâte qu'avaient eu certains sociologues à affirmer scientifique leur discipline, ou du désintérêt d'autres pour la question. Le vieux Weber me semblait être le seul à se situer dans la bonne direction, quand il remarquait qu'il n'était pas donné à toutes les sciences d'atteindre la maturité. Mais il me semblait qu'il était possible d'aller plus loin.

J'ai donc commencé à parler de «préalables », qui étaient à examiner avant de pouvoir définir le type de scientificité des sciences sociales ${ }^{2}$. Je m'étais convaincue, au cours des années, de l'importance de certaines notions pour caractériser les sciences sociales, telles que je les voyais exercer autour de moi: ainsi m'était apparue l'importance de la subjectivité, qui conduisait à l'idée d' «inhomogénéité des connaissances»; la reconnaissance de l'existence d' «identités épistémiques »; la notion d" «épistémologie éclatée»; la «non-séparation entre savoirs savants et savoirs profanes » (d'où le terme général de «savoir social»)...

Parmi ces préalables, il y a le problème de l'éthique. Bien sûr, depuis les débuts de la discipline - issue en fait des «sciences morales»-, les chercheurs se sont penchés sur la question de la morale et de la société. On sait que Durkheim et Weber, pour en revenir à eux, ont eu des points de vue différents là-dessus. Mais le problème déborde ces positions de type sociologique. De fait, tout travail de sociologie, tout travail de connaissance sur la société, est une intervention sociale, une intervention dans la société, avec des effets plus ou moins importants et plus

Cette fois encore, j'examinais ces disciplines du dehors. D'autres chercheurs, qui s'identifient plus que moi à leur discipline, travaillent à faire ressortir les formes habituelles de l'explication (tels Berthelot ou Passeron). 
ou moins connus. De nouveau, la situation est différente de celle qui prévaut dans les sciences exactes: on y trouve d'abord une «éthique interne» à la communauté, où rigueur, honnêteté, sont attendues dans le travail de connaissance, puis une «éthique externe», qui se préoccupe des utilisations qui pourraient être faites des découvertes. On sait que la radioactivité peut servir à la guerre atomique ou aux soins des malades. Le fait que la biologie actuelle ne participe pas de ce schéma, puisque les problèmes éthiques se posent avant même les découvertes, provient de ce qu'il s'agit d'une science portant sur l'homme, et refusant donc, éventuellement, au nom de principes éthiques, une manipulation que l'on accepte dans le cas d'objets.

Le cas des sciences sociales s'apparenterait à celui de la biologie. D'où un début d'intervention de comités d'éthique, dans des expériences psychologiques, par exemple.

L'article où j'essayai de mettre au clair toutes ces pensées s'avéra, une fois encore, très difficile à écrire. Il s'agissait de recenser les «défis » particuliers que posait la question de la connaissance «scientifique» du social, qui me semblaient à la fois d'ordre éthique, épistémologique, et social (dans la mesure où il s'agissait d'une intervention dans la société). Cette étape une fois franchie, je n'étais pas encore satisfaite. J'avais toujours l'impression de ne pas être parvenue au fond de la question.

Cela fut l'affaire de mon travail de cet été. Et cette fois, ce sont des «axiomes » que j'ai voulu trouver. L'axiome est supposé être le point de départ de la réflexion mathématique, mais il s'agit de bien choisir ce point de départ. Il est comme un résumé de ce qu'il pourra engendrer. Il est donc aussi l'aboutissement d'un long travail de recherche de l'essentiel.

Et cette fois encore, ce fut un travail ardu que d'arriver à ce dépouillement maximal, qui signifiait une plongée dans les profondeurs de la réalité, pour en trouver les «bases». J'ai alors eu l'image du cyclone, où la violence de la tourmente était d'autant plus forte qu'on s'avançait vers le centre, cet œil où en définitive, régnait le calme, une fois qu'on a pu l'atteindre.

\section{RÉFÉRENCES DES TEXTES ÉVOQUÉS}

1986- «Quelques repères sur les savoirs sociaux. Pour une expérience de communication », dans Le Sujet et l'Objet. Implications, Paris, Ed. du CNRS, pp. 175-203.

1991- «Préalables pour une certaine scientificité des sciences sociales », exposé au séminaire Éthique et Épistémologie des sciences sociales.

1993- Contribution au débat: «CNRS : sécession des sciences de l'homme?» Le Débat, 76, sept-oct, 215-218.

1994- «Which Scientificity for the Social sciences?», World Futures, 42, 133-143.

1998- «Sur quelques défis d'ordre épistémique, moral et social des Sciences de 1'Homme et de la Société» Les «travaux du GEMAS», n³, Paris, mai 1999. à paraître dans Jean-Paul Terrenoire: La responsabilité des scientifiques, 1'Harmattan, 2001. 


\section{II. ÉNONCÉS ET COMMENTAIRES}

On a deux séries d'axiomes, distinguées par les lettres A, pour les sciences exactes et $\mathrm{B}$, pour les sciences humaines. Chaque premier axiome porte sur les caractéristiques de la réalité qu'il s'agit de connaître, ce sont, en quelque sorte, des axiomes ontologiques, qui conditionnent les modes de la connaissance. Chaque second (et dernier) axiome porte sur l'action de connaître, avec ses succès et ses limites, ce sont des axiomes épistémologiques.

On commence par les sciences exactes, pour les raisons évoquées et parce que la situation y est effectivement plus simple.

\section{Deux axiomes pour la réalité physique et mathématique et sa connaissance}

Axiome A1. La réalité extérieure, mathématique ou physique, existe indépendamment de moi et possède des structures fortes.

\section{Commentaires:}

- «possède des structures fortes », et non pas « est fortement structurée ». La deuxième formulation impliquerait une structuration partout, la première implique seulement des structurations partielles, celles qui ont trait, par exemple, aux particules élémentaires, ou au Tableau de Mendeleieff, et non pas les structurations faibles qui affectent ce qu'il est convenu d'appeler les systèmes complexes (ex: la météo) (lesquels peuvent faire l'objet de modélisations mathématiques fortes, par ailleurs). C'est en raison de l'existence de ces structurations fortes dans les sciences de la nature (physique et biologie) qu'on parle de sciences exactes. La structure a à voir avec ce qui peut être bien défini, donc avec l'exactitude.

- La structure implique «l'objet», cet élément constitutif des sciences exactes, bien défini, manipulable, (d'où la possibilité de l'expérimentation) qui permet d'arriver à l'objectivité de la connaissance. Elle implique cet autre élément constitutif, la «causalité forte», qui permet l'enchaînement des découvertes, des questions, et de nouvelles découvertes. C'est ce qu'on nomme la «cumulativité ».

- Les mathématiques, créées par l'homme, possèdent également une structuration forte. De fait, elles, elles sont fortement structurées. C'est la raison pour laquelle elles ont pu s'appliquer à la physique et bénéficier des développements de la physique. Avant la mesure des champs (géométrie), la numération (arithmétique) avait d'ailleurs tout aussi pu s'appliquer aux hommes (dénombrement des populations, cf. Bible), qu'aux troupeaux, aux arbres, etc....

Passons à présent au deuxième (et dernier) axiome: 
Axiome A2. Je m'efforce, et je peux, jusqu'à un certain point indéterminé, découvrir ces structures

\section{Commentaires:}

- Cet axiome, après le premier qui m'avait déjà introduit/e, se fixe sur moi, qui écris ceci, qui pense ceci, mais qui représente aussi un moi générique, celui de l'espèce humaine, sans qui la connaissance, et donc les sciences exactes, n'existeraient pas.

Le statut du «je», dans les sciences exactes, est spécifique. Je suis la personne qui cherche à connaître, seule et avec d'autres. J'y mets mon énergie, c'est un travail loin d'être donné. Il faut, d'une part, «imaginer vrai», comme disait Feynman, et ensuite faire la preuve de ce qu'on avance. De fait, en raison de l'objectivité possible ici, mon «moi» se subsume, en principe, dans celui de l'humanité physicienne, découvrant des «lois» universelles. La réalité pragmatique de la recherche en train de se faire est certes une entreprise humaine souvent bien plus confuse, mais cette confusion ne doit pas faire oublier le principe très simple, en l'occurence, qui a permis cette extraordinaire cumulativité de résultats inattendus, aboutissant à ces merveilles techniques qui sont devenues si banales que nous ne nous en émerveillons plus - quand elles ne nous font pas peur pour leurs possibles applications dangereuses, voire, destructrices.

- Contrairement à une certaine épistémologie qui a le vent en poupe actuellement, je maintiens la séparation entre l'objet et le sujet, déjà signifiée dans le premier axiome. La réalité résiste, on ne peut pas tout en faire.

Par contre, contrairement au scientisme qui oublie le sujet de la connaissance, je le réintroduis, comme une part essentielle, vivante, pensante, fantasmante, etc. C'est dans la ligne de la définition de la connaissance comme «interaction entre un sujet et un objet de connaissance», que j'ai introduite plus haut.

- Je m'efforce: signale l'effort gigantesque déployé par notre société pour développer ces connaissances. Je peux: signale la réussite étonnante, qui était loin d'être évidente a priori, de cette démarche.

- Enfin, je tiens à introduire les limites éventuelles de la connaissance. Contrairement à certains physiciens qui, périodiquement, pensent que la physique est terminée, que toutes ses lois fondamentales ont été découvertes (c'était ainsi le cas à la fin du 19ème siècle après l'étonnante synthèse de Maxwell, et c'est le cas aujourd'hui pour un Weinberg qui croit que l'on n'est plus loin du modèle définitif), un scepticisme, à moins que ce ne soit un certain type de sentiment de religiosité, me conduit à tempérer des enthousiasmes prématurés et à parier sur une impossibilité fondamentale d'arriver au bout de la connaissance, ce qui n'empêche pas le désir qu'elle soit poursuivie autant que faire se peut. Mais les énormes besoins d'argent que nécessitent des appareillages toujours plus puissants pour percer des mystères toujours plus près de l'infini (petit ou grand), me donnent à penser que l'infini restera hors de notre portée. Telle quelle, cette reconnaissance de limites à la connaissance apparaît aussi comme un garde-fou contre le fantasme de toute-puissance d'un certain scientisme. 


\section{Deux axiomes pour la réalité sociale et psychique et sa connaissance.}

Axiome B1 : La société humaine est un système complexe qui est en perpétuelle auto-création et dont je fais partie.

Axiome B2: Dans mes tentatives variées de connaissance des réalités sociales et psychiques, je suis limitéle par des aveuglements et des résistances qui font profondément partie de moi-même.

Il y a en commun entre les deux systèmes la présence d'un sujet désireux de connaître, le mieux possible, des «objets de connaissance».

Dans les deux cas, le sujet de la recherche de connaissances, c'est moi, d'une part, un moi générique de l'humanité, qui vise à une connaissance objective, mais aussi, d'autre part, cette personne-là que je suis, et qui prend des risques et doit assumer ses responsabilités dans l'énoncé de ses connaissances. Or, le jeu entre objectivité et subjectivité des connaissances s'avère différent dans les deux cas.

- Je fais partie de la société. Ceci introduit des éléments tout à fait nouveaux.

Dans le premier cas, le sujet de la recherche est en quelque sorte divisé: il est réduit au moi logique, curieux, rationnel, intelligent, intuitif, certes, mais critique...Il vise à la connaissance objective, qui se trouve être universelle. Il fonctionne par rapport à l'hypothèse bien définie qu'il va falloir prouver; ou encore, par rapport à la découverte, qu'il va falloir reproduire. Ce n'est pas que ses affects aient disparu pour autant. Ce sont eux qui produisent sa motivation au travail: curiosité, ambition, désir de reconnaissance, les émotions ne manquent certes pas, qui accompagnent les étapes du travail: joie intense d'avoir réussi, déception d'avoir échoué, malaise de s'être trompé. Il y a loin de cette réalité vibrante, passionnée, à l'image du savant impassible qui est parfois donnée. Mais il n'en demeure pas moins que toutes ces émotions, ces passions, sont au service de la connaissance objective. La subjectivité du scientifique est concentrée sur son caractère de scientifique.

Pour qui cherche à connaître la société, il y a plusieurs manières de le faire. Le seul fait d'y appartenir en procure une connaissance qui n'est pas toujours, comme semble le dire la méthodologie orthodoxe, fausse. C'est ce que j'avais appelé le savoir «senti», qui se situe avant la verbalisation.

Pour qui vise à une certaine prise de conscience, ce qui est un minimum pour tout acte de connaissance, on peut choisir plusieurs postures pour la connaissance sociale, le terme de posture impliquant un choix, une intentionnalité.

L'engagement dans la cité, l'action, procure certainement de la connaissance, et, par ailleurs, utilise la connaissance.

Mais les sciences sociales tiennent à se caractériser par une distanciation (qui tiendra lieu, pour beaucoup, de «scientificité»). Cette distanciation peut prendre la forme de la réflexion, ou encore du travail sur documents. Une autre forme est celle de la construction d'observations chiffrées (les «statistiques», les «sondages», les diverses «mesures» qui sont socialisées, utilisées par la société, comme le QI, par exemple). On peut également aller «sur le terrain», selon des modalités diverses: observation plus ou moins participante, accompagnement psychothérapeutique, intervention psychosociologique, recherche-action. 
La séparation entre le sujet épistémique, celui qui cherche la connaissance valide, et le sujet psychique et social, n'est pas aussi simple que dans le cas des sciences de la nature.

On reviendra plus tard sur ces difficultés. Pour l'instant, je voudrais considérer le sujet épistémique ayant fait le choix de la distanciation et examiner quelques obstacles spécifiques qu'il ne manque pas de rencontrer dans son travail.

- La complexité est une notion utilisée actuellement dans plusieurs domaines de la connaissance - mathématiques, physique, sociologie - avec des définitions propres à chacun de ces domaines, très précises dans les deux premiers cas. Elle se distingue, bien entendu, de la simplicité, mais aussi de la complication: dans ce dernier cas, on peut avoir l'espoir d'arriver à démêler l'écheveau, car, derrière les nœuds et les embrouillages qui se présentent en premier, on conçoit - ou l'on postule - l'existence d'une structure. C'est pourquoi la météorologie, dans ce sens, n'est que compliquée, et non complexe, on en connaît tous les éléments de base, c'est dans leurs combinaisons multiples qu'on se perd ${ }^{3}$.

Dans les sciences sociales, je mettrai en avant deux propriétés où jouent cette «complexité»: l'absence de causalité forte et l'absence d'objets bien délimités, propriétés qui s'accompagnent et se renforcent en quelque sorte.

1. l'absence de causalité forte - provient de la non transitivité, en règle générale, d'une corrélation. Il est possible, en effet, d'établir des corrélations significatives entre deux «dimensions». Mais ces corrélations sont faibles, et l'enchâ̂nement des résultats s'essouffle rapidement. Il n'y a pas cette cumulativité qui a permis le développement de la physique ou de la biologie, grâce à leurs résultats «durs».

1bis. Pourtant, il existe bien des corrélations entre diverses variables. Des mini-structures, des schèmes causaux, des régularités, permettent, localement et temporairement, de proposer des modèles bien définis qui ont une certaine pertinence et validité. C'est le cas en économie, bien sûr, mais encore, en psychosociologie: par exemple, les «jeux» définis par Eric Berne montrent des réactions constantes à certaines situations. C'est au niveau de la transitivité des résultats que la construction ne pourra aller très loin.

2. l'impossibilité d'isoler réellement les parties. Contrairement aux «objets physiques », on ne peut manipuler des parties de la société ou du psychisme (dans ce dernier cas intervient l'éthique). C'est donc à travers des discours qu'on décrira l'«objet», celui que la méthodologie nous engage à «construire», c'est-à-dire à préciser, à délimiter. Car pour connaître il faut bien décomposer, ce que recommandait Descartes en son temps, avec la règle de remettre ensemble les parties

On peut encore placer sous le chapeau de cette notion l'existence d'une réalité «intérieure », que l'on peut qualifier aussi de «virtuelle» ou de «potentielle», dans la mesure où elle peut éventuellement se manifester «à l'extérieur». Appartient en effet à l'être humain un ensemble sous-jacent à son comportement visible de désirs, valeurs, préférences, imaginations, représentations, rêves éveillés ou non, intentions...Pour l'exploration de ce monde intérieur, et en y rajoutant la notion d'inconscient, la psychanalyse a instauré un «dispositif» spécifique de connaissance et d'interaction. 
ensuite, ce qui, dans notre cas, n'est guère faisable: le tout est plus que les parties, comme il est coutume de dire.

Il n'est que de comparer la dimension des écritures dans les sciences sociales et psychiques et dans les sciences exactes pour voir à quel point les premières sont tributaires du discours, alors que dans les secondes, le discours est réduit au minimum, la description de l'expérience faite et du résultat obtenu. Cette prégnance du discours fait que les sciences sociales continuent à se situer du côté de la culture littéraire, avec ses effets de rhétorique, où les connaissances vastes sont un atout puisqu'il s'agit d'objets complexes, et l'art de savoir en parler un avantage puisqu'il faut convaincre par le discours; tandis qu'une connaissance réduite et un discours resserré peuvent suffire pour la connaissance d'un objet bien délimité.

Il en résulte une hétérogénéité des connaissances et de leurs modes. Les modes d'accès à l'objet complexe sont en effet divers. Les éclairages, points de vue, sont multiples. Les perceptions peuvent être différentes: pour l'un, tel phénomène est accessoire, mineur, pour l'autre, il est essentiel. Dans la délimitation même de l'objet, des désaccords apparaissent: on trouvera malvenues certaines comparaisons, on accusera d'amalgame celui qui confondra deux éléments que, soi-même, on distingue nettement. On peut se placer à des niveaux d'appréhension plus ou moins abstraits, plus ou moins fins également: du pragmatique «ici et maintenant» à la visée à long terme; de l'approche qui se contente de la réalité immédiate, à celle qui cherche les réalités sous-jacentes plus profondes; de celle qui cherche, au-delà des premières apparences, des phénomènes cachés.

Les efforts de définition quantitative des statisticiens tentent de pallier au caractère complexe des objets, en les fixant, mais, de façon volontariste, et, de fait, sociale: on tente de suivre les évolutions de ce qui parait important dans la société.

En l'absence d'objet défini, et en la présence de situations toujours complexes, interviennent, dans la visions des choses, des philosophies de base non explicitées, et non explicitables complètement. L'effort d'explicitation fait perdre au sociologue son caractère de sociologue, il devient philosophe, approfondissant certains thèmes, au détriment d'une vue globale.

Correspondant à toutes ces variations possibles, les styles utilisés sont aussi très divers: le style dit impressionniste s'efforce de coller à la richesse de la diversité de la réalité, par touches successives d'où tout modèle rigoureux est exclu. Il est évidemment proche du style littéraire, avec lequel, comme on sait, on peut parvenir à décrire de façon très pertinente le genre de réalités dont il s'agit dans la connaissance de l'humain. De la même façon, tout travail d'approfondissement des concepts, toute vision large se rapproche d'un style de type philosophique. Le style quantitatif, quant à lui, portant sur les statistiques, sera celui qui pourra se donner l'aspect le plus scientifique, sans pourtant qu'un effort de réflexion sur la constitution - qui est aussi construction - des données puisse être évité.

Trop souvent, ces différences d'approche prétendent à être les seules valables, alors même qu'il serait plus honnête de reconnaître les sensibilités propres qui portent vers chacune d'entre elles, voire, font rejeter d'autres. Il faudrait pour cela la prise de conscience et la reconnaissance de l'identité épistémique qui caractérise chacun d'entre nous.

En conclusion, on peut parler d'une épistémologie éclatée, puisque les modes de connaissance sont divers, et se juxtaposent sans vraiment donner lieu à une véritable confrontation ou sans jamais se compléter. 
La prise en compte, dans l'axiome B1, de la notion de complexité, prise dans ce sens, un sens fort, signifie bien l'adoption d'une philosophie où l'on pose les limites de la connaissance de l'être humain et de ses sociétés.

Comparons alors avec l'axiome où intervenait l'impossibilité de la connaissance ultime, pour la réalité physique. C'est le mot «ultime», ici, qui va marquer la différence. Cette position intervient au bout d'un processus de connaissance qui se développe, à l'infini d'une chaîne structurée de connaissances toujours plus précises. Or, cette cumulativité qui fait la spécificité des connaissances des sciences exactes, n'existe pas dans les sciences sociales. Il y a bien sûr accumulation de connaissances, développement de théories, mais c'est comme si on en restait à un niveau de surface, abordé par les divers points de vue, sans que des résultats durs, certains, quasiment définitifs, puissent permettre de continuer la construction structurée d'un savoir.

Les divers savoirs, bien réels, se chevauchent, ils ne s'ordonnent pas. On reste dans des plans de connaissance de nature différente, où l'approfondissement de ces connaissances n'a pas le même sens. Dans le cas de la physique, il s'agira d'une approche toujours plus minutieuse de l'objet, avec des différences d'ordre de grandeur allant des mesures dites astronomiques aux mesures toujours plus fines de la réalité subatomique, avec des cohérences pour passer d'un niveau à l'autre. Dans le cas des sciences sociales et psychiques, un approfondissement peut travailler une notion, par exemple, mais cet approfondissement de nature philosophique peut perdre de sa pertinence pour la solution de problèmes immédiats. Les niveaux de la sociologie, de la psychosociologie, de la psychologie, de la psychanalyse, à la fois s'interpénètrent partiellement tout en ayant à distinguer leurs logiques spécifiques. Les théories sociologiques peuvent être à portée différente (Merton) avec leurs pertinences respectives. De même, la description d'événements historiques peut se faire selon la longue durée ou s'enfoncer dans le pointillisme de l'histoire locale.

Dans le cas des sciences exactes, la rigueur allait de pair avec la pertinence, ces deux notions se distinguent ici: certains résultats menus, aussi rigoureux que possibles, semblent sans pertinence pour qui est à la recherche d'une vision plus large ${ }^{4}$.

On pourra évoquer ici, au lieu de la cumulativité des sciences exactes, une évolution des idées dont on peut faire l'histoire, mais sans qu'il existe cet ordonnancement qui permettait la cumulativité des résultats.

L'auto-création à la fois apporte de nouvelles limites dans le champ de la connaissance objective et ouvre des possibilités dans le domaine de la liberté, et donc, de l'éthique.

La société se crée continuellement, et nous-mêmes également, à partir de la connaissance acquise, et en fonction des buts poursuivis. On rappellera que la liberté n'implique pas qu'il n'existe aucune régularité, aucun déterminisme, même. Elle représente une expérience de sentiment, ou sensation, l'impression d'une ouverture, de choix possibles, même si, après coup, on peut «expliquer» ces choix, autrement dit, les lier à d'autres réalités et en retrouver la logique.

4 C'est le reproche souvent adressé aux résultats de la psychologie expérimentale, qui singeraient la forme scientifique, sans arriver pourtant à une véritable cumulativité. 
L'évolution non déterminée a priori d'un système en rend plus difficile l'étude - mais en même temps, peut-être plus nécessaire encore, pour tenter, au moins, quelques prédictions, quelques guidages, pour ne pas rester dans le chaos total.

Dans les sciences exactes, la notion d'évolution existe bien, et peut même donner lieu à des modèles quantitatifs forts. Mais le temps de la science exacte peut être en quelque sorte figé, de façon à pouvoir reproduire, vérifier, recommencer autant qu'il est nécessaire une expérience donnée, un résultat obtenu, la réalité de l'évolution annoncée. Dans le cas des sciences sociales, les évolutions dépassent notre temps de vie. Les échelles de temps sont inversées.

- l'auto-création accompagne le phénomène d'appartenance à la société, appartenance des sociologues, et donc, de moi-même. Toute étude sociologique est un événement qui appartient à la société, de plus ou moins grande importance, avec des effets plus ou moins marqués. Tout ce qui nomme, classe, commente, reconnaît, fait apparaître, décrit... est une intervention sociale. Le plus souvent, on peut dire que tout travail de sociologie est à la fois symptôme et agent, symptôme, il apparaît dans une situation donnée, agent, il participe de l'évolution de cette situation.

Les sciences sociales sont ainsi constituées à l'intérieur même de la société, en une espèce de corps intermédiaire, où à la fois on agit et à la fois on tient à garder une certaine distance qui permettrait plus d'objectivité. Mais la frontière est loin d'être étanche entre les savoirs profanes, ceux que tout un chacun a sur la société, du fait qu'il y participe, et les savoirs savants, ceux qui sont reconnus par la cité académique. La pertinence, la justesse, n'est pas toujours du côté de ces derniers. L'expertise est ainsi très différente de celle qui a lieu dans les domaines mieux définis, où les options de base peuvent être explicitées, et les non-connaissances plus facilement admises, parce que plus nettes.

- l'appartenance à la société implique que les problèmes éthiques se situent avant, pendant, et après le travail de connaissance: avant, parce que la mise en lumière d'une portion de la réalité est déjà une intervention. Pendant, parce que, si l'on travaille directement avec des personnes, des limites à l'intervention, et donc à la connaissance, doivent souvent être prises en compte, comme le savent bien, tout particulièrement, les psychanalystes. Ensuite, parce que les effets du travail se produisent dans la société.

Mais il existe aussi une implication proprement épistémologique à cette situation. C'est ce qu'ont bien vu les premiers sociologues allemands, en introduisant le terme de «compréhension »: du fait que je participe à la société, que je suis un être humain, je peux m'identifier à un autre être humain, et le «comprendre» dans une situation de connaissance psychologique. D'autres termes peuvent être utilisés, pour une interaction très spécifique, et qui défie les règles habituelles de l'explicitation de type scientifique: empathie, croisement d'inconscients, résonances, «senti »....

C'est ce caractère totalement humain de celui qui cherche à connaître d'autres êtres humains qui conduit à l'axiome B2.

Cet axiome pointe, d'une part, la variété des modes de connaissance, et d'autre part, leurs limites intrinsèques.

Dans le cas de la physique, l'axiome de non-connaissance ultime se réfère à l'infini (dans le temps) d'une démarche dont, à tel instant fini, rien ne dit que nous 
avons à nous arrêter, sinon pour des raisons financières, percer les énigmes de l'infiniment petit ou de l'infiniment grand devenant sans doute un jour trop cher pour nos sociétés, qui peuvent avoir d'autres priorités par ailleurs.

Dans le cas de la connaissance de l'humain, il y va de la relation à l'autre et d'abord, de l'éthique, qui veille à ce que le travail de la connaissance et ses effets ne soient pas de l'ordre de la manipulation, ou de l'instrumentalisation de l'être humain, conduisant à introduire éventuellement des réserves dans la connaissance ${ }^{5}$. La reconnaissance de la nécessité de la suspension de jugement, de la « non-connaissance», est importante, et irait dans le sens de la philosophie orientale, une philosophie qui justement n'a pas développé de science exacte.

Mais cet axiome est aussi celui de l'impossibilité de la connaissance réflexive totale, qu'on pourrait aussi exprimer, de façon imagée, de la façon suivante: Les yeux sont placés de telle sorte qu'ils ne peuvent se voir directement ${ }^{6}$.

Est ainsi introduite l'impossibilité de se connaître complètement, autrement dit, l'existence inévitable d'aveuglements et de dénis de connaissance, un résultat qui a été introduit avec force par la psychanalyse.

Cette reconnaissance de l'existence de points aveugles, de résistances (plus ou moins conscients) outrepasse celle des simples résistances intellectuelles du champ A, celui des sciences exactes, en raison de leur pouvoir à affecter profondément.

L'affect qui intervient dans la connaissance de l'humain le fait sous deux registres : connaissance, disons, intuitive (compréhension, empathie...), et méconnaissance.

2.

\section{EXISTE-T-IL UNE SCIENCE POST-MODERNE? LA SIGNIFICATION DE L'«AFFAIRE SOKAL»*}

\section{L'«AFFAIRE SOKAL»}

\section{Le canular}

Alan Sokal est un professeur de physique théorique d'une université américaine. Il est agacé par les absurdités qui sont très souvent écrites à propos de la science par des auteurs appartenant au courant intellectuel et idéologique dit «post-moderne »: il était de bon ton de dénier à l'approche scientifique sa spécificité en mettant l'accent sur ses aspects sociaux et en montrant qu'elle ne différait pas d'autres activités sociales; la notion de croyance, par exemple, vient rempla-

5 Cf J.Feldman et Ruth Canter Kohn (coord.) L'éthique dans la pratique des sciences humaines: dilemmes, Paris, L'Harmattan, 2000.

6 Le subterfuge du miroir ne fait que limiter cette impossibilité de se voir complètement, et introduit par ailleurs le biais de la symétrie droite-gauche.

* Ce texte a d'abord fait l'objet d'une version anglaise parue dans Philosophy and Social Action, 1999, 25(3), 19-30. 
cer l'idée de la preuve. Sokal voit dans les activités de ce courant une menace pour la qualité de la pensée, en raison du bas niveau de ses exigences intellectuelles. C'est cette absence de rigueur minimale qu'il a voulu illustrer.

Il écrit un article sur la science où il utilise le jargon post-moderne habituel. Le titre de l'article est: "Transgresser les frontières: vers une herméneutique transformative de la gravité quantique $»^{7}$. C'est en lui-même un petit chef-d'œuvre en condensé. Il contient plusieurs allusions importantes à la pensée post-moderne. L'idée de transgression évoque une position critique, révolutionnaire, envers la science et la société. Le refus des frontières dénote l'opposition à l'orthodoxie intellectuelle, et permet, en particulier à des non-scientifiques de parler de la science. Transformative donne de nouveau l'idée d'un changement. L'herméneutique s'oppose au positivisme, la philosophie dominante aux USA. Pour finir, la gravité quantique est un des champs les plus récents de la recherche en physique, qui s'efforce d'unifier la théorie de la gravitation et la mécanique quantique. Certains aspects de la mécanique quantique sont cités régulièrement par le postmodernisme, ils sont censés illustrer le fait que la physique moderne reposerait sur l'incertitude et la subjectivité à travers l'interaction entre le chercheur et son objet de recherche. La relativité et la mécanique quantique apparaissent ainsi comme relevant d'une physique post-moderne, parce qu'elles soulèvent des problèmes au sujet de nos conceptions habituelles de la réalité. Il s'agit évidemment d'une méprise totale de la situation. Les deux champs, quelque soient par ailleurs les problèmes philosophiques qu'ils soulèvent et qui peuvent éventuellement diviser les physiciens, ont un impressionnant pouvoir de prédiction, ce qui veut dire qu'ils sont capables de fournir une image étonnamment précise de la réalité physique, comme Sokal l'expliquera plus tard dans son livre (voir plus loin).

Ainsi, le titre de l'article fait appel à des idées à la mode, qui sont idéologiquement sympathiques aux éditeurs de la revue Social Text, auxquels il est envoyé. Il semble se situer sur les bords avancés à la fois de la philosophie et de la recherche la plus pointue en physique.

L'article développe des critiques de la science et de la physique moderne, en utilisant lourdement les thèmes habituels au post-modernisme et propose, évidemment, une nouvelle physique. Il contient un nombre considérable de citations (exactes) venues des guides intellectuels coutumiers du post-modernisme.

L'article est publié dans un numéro spécial de Social Text consacré à «Les guerres de la science ${ }^{8}$. Plusieurs semaines plus tard, Sokal révèle, dans une autre revue, qu'il s'agit d'un canular. ${ }^{9}$

\footnotetext{
Alan Sokal, 1996, «Transgressing the boundaries: Toward a transformative hermeneutics of quantum gravity ». Social Text, 46/47, 217-252.

8 Ce numéro constituait une réponse à plusieurs attaques qui avaient été lancées précédemment par des scientifiques contre les vues post-modernes sur la science. Par exemple: Gerard Holton: Science and Anti-Science, 1993, Cambridge and London, Harvard University Press. - Paul R.Gross and Norman Levitt, 1994, Higher Superstition. The Academic Left and its Quarrels with Science, Baltimore and London, The John Hopkins University Press. - Gross, Paul R., Norman Levitt et Martin W. Lewis (editors), 1996. The Flight from Science and Reason. Annals of the New York Academy of Sciences, 775.

9 Sokal, Alan, 1996, «A physicist experiments with cultural studies». Lingua Franca 6(4) (May/June 1996), 62-64.
} 


\section{Le livre L $^{10}$}

L'effet du canular est immense, et largement inattendu. La polémique est intense dans la presse américaine, puis, très bientôt, dans la française. Il se trouve en effet que la plupart des maîtres intellectuels «post-modernes » qui sont cités par Sokal sont français. Le psychanalyste Jacques Lacan affectionnait d'utiliser les mathématiques pour la description de la névrose; le philosophe Jacques Derrida a popularisé la notion de déconstruction, Baudrillard et Lyotard celle de postmodernisme; le sociologue Bruno Latour prétend que la science est «sociale de part en part»; la linguiste Julia Kristeva a tenté de donner un modèle mathématique de la poésie; la féministe Luce Irigaray a plaidé pour une science féminine.

Surpris par le succès de sa plaisanterie, Alan Sokal tente d'engager un dialogue réel et d'expliquer son canular. Avec un collègue belge, Jean Bricmont, qui est intéressé dans la philosophie de la science, il écrit un livre. Là, les auteurs s'efforcent d'expliquer de façon très pédagogique les différentes erreurs et méprises que ces intellectuels français célèbres - mais aussi d'autres - ont faites quand ils se sont référés à des thèmes de physique et de mathématiques à la mode ( tels que la relativité, la mécanique quantique, le chaos, le théorème de Gödel, la théorie des ensembles, etc.). Ils discutent aussi les thèses relativistes sur la science de quelques philosophes américains et de Latour. Pour terminer, ils dévoilent les différentes astuces du canular et soulignent ses aspects rhétoriques.

Leur but est d'engager la lutte contre ce qu'ils considèrent être des «impostures intellectuelles». Ils visent à restaurer le «sens commun», la simplicité de l'expression, la clarté de pensée, qu'ils considèrent en danger en raison de la séduction exercée par les idées à la mode, brillantes et confuses.

Le livre soulève à son tour une violente discussion, à la fois dans les journaux et dans la communauté intellectuelle ${ }^{11}$. Une partie considérable de la discussion a également cours sur Internet. Beaucoup se sont réjouis de la dénonciation mais beaucoup d'autres se sont sentis offensés et l'ont reprochée aux auteurs. A travers les confrontations passionnées, on a vu se poser des questions tout à fait intéressantes et utiles sur la place et les modes du travail intellectuel dans la société moderne. La signification de l'»affaire» peut être le mieux appréciée si on la replace dans son contexte socio-historique.

\section{LE CONTEXTE SOCIO-HISTORIQUE}

\section{L'accroissement de la classe moyenne et l'extension de la science}

Pendant une longue période, l'éducation a été réservée à une minorité privilégiée. Mais les sociétés modernes dépendent de leurs capacités à développer des connaissances techniques et scientifiques. Les progrès techniques ont contribué à

\footnotetext{
10 Alan Sokal et Jean Bricmont, 1997, Impostures intellectuelles, Paris, Editions Odile Jacob. 1999, Paris, Le livre de poche.

11 J. Feldman, 1999, «L'AAffaire SOKAL': Un épisode de la méconnaissance entre cultures ». L'Année Sociologique, 49, 245-270.
} 
la réduction du nombre des personnes engagées dans les artisanats traditionnels comme dans l'agriculture. De plus en plus d'enfants sont engagés dans une éducation à long terme, ce qui augmente considérablement les classes moyennes. En France, le but officiel est de faire atteindre à $80 \%$ d'une classe d'âge le niveau des études supérieures. On parle désormais d' «éducation de masse».

La science a aussi subi beaucoup de changements. En raison de ses succès, elle s'est étendue à de nombreux nouveaux domaines, au point de tenter d'englober tous les aspects de la connaissance. Mais tous ces nouveaux domaines ne sont pas susceptibles de produire des résultats forts et décisifs comme cela a été le cas pour la physique et la biologie au cours des trois derniers siècles. Lorsque la production d'articles «scientifiques» décide de la carrière de beaucoup de ces personnes issues des classes moyennes, on peut voir sans surprise fleurir de nouvelles « sciences », où il n'est plus tellement question d'arriver à des résultats réels qu'à pratiquer diverses sortes d'exploration «heuristique». Ces nouvelles sciences prennent l'aspect social de la science. Le physicien et philosophe François Lurçat les a nommées les «social-sciences»: les chercheurs travaillent ensemble, ils créent des «revues scientifiques», organisent des colloques, demandent des subventions, etc.

Parmi ces nouvelles sciences, on peut placer les sciences humaines et sociales, dont le but est d'apporter une connaissance qui se veut scientifique sur les gens et les sociétés. Elles se disputent beaucoup au sujet de leurs méthodes. Le point intéressant est que, parmi elles, seule l'économie a obtenu le statut prestigieux du droit au prix Nobel. C'est à la fois la discipline sociale qui travaille le plus avec des méthodes mathématiques, d'où son aura de scientificité, mais c'est également celle qui est la plus centrale dans cette idéologie dominante que représente le libéralisme.

\section{La critique de la société}

La transition à ce nouveau stade de la société, de l'éducation et de la recherche a donné lieu aux mouvements d'étudiants des années soixante et soixante-dix. Les étudiants qui, autrefois, auraient considéré qu'ils accédaient à des statuts privilégiés dans la société, s'aperçurent que ce n'était plus le cas. Ils étaient en train de devenir des sortes de prolétaires intellectuels, sans véritable liberté de développer leur personnalité et leurs propres pensées. Et ils se sentirent solidaires des peuples opprimés dans le monde.

La nature critique du mouvement étudiant a été extrême. Elle englobe toute la vie: privée, avec la demande de la libération sexuelle et le mouvement de libération des femmes - et publique. La société de consommation est dénoncée, ainsi que ses aspects de rationalisation. La science, une des institutions dominantes de la société, subit aussi, et pour la première fois de l'intérieur même, un examen critique minutieux; le mouvement écologique dénonce les retombées néfastes des industries chimiques et nucléaires. Plus tard, des scandales largement médiatisés - comme les transfusions de sang contaminé ou la maladie de la vache folle - soulignent le manque éventuel de fiabilité des expertises.

On doit encore souligner un autre point: jusqu'ici, la critique de la société avait été conduite à partir d'un point de vue politique. Ceux qui dénonçaient le capitalisme proposaient une autre société, basée sur le socialisme. Mais il fut clair, 
dans les années soixante, que les diverses expériences vers le socialisme avaient échoué. Quelques années plus tard, la chute du mur de Berlin allait illustrer de façon spectaculaire ces échecs. Après l'effondrement des états socialistes, il ne resta qu'un seul modèle pour la société moderne, le capitalisme, avec ses possibilités de liberté relative. Les mouvements des années soixante et soixante-dix développèrent l'idée que la politique recouvre tous les aspects de la vie.

Tout se passa comme si l'absence de l'idée d'un autre monde possible laissa les jeunes idéalistes avec la seule possibilité de jouer à la critique radicale à l'intérieur de leurs propres sociétés et de leurs propres cercles, en particulier l'Université. C'est ce qui pourrait expliquer l'esprit hypercritique du «post-modernisme».

\section{LE POST-MODERNISME}

N'étant pas une philosophe professionnelle, je ne m'engagerai pas ici dans le débat philosophique sur le post-modernisme. Il faut noter que beaucoup de ceux qui sont considérés par Sokal comme post-modernes rejettent ce label. Il est évident qu'un philosophe subtil refusera toujours de voir sa pensée résumée en une formule. On peut se souvenir à ce point que Marx a dit qu'il n'était pas marxiste.

Ce que je voudrais évoquer ici, c'est l'atmosphère générale qui suivit le mouvement de critique de la science et de la société, quand il devint une tendance dominante de la critique de l'establishment intellectuel, visant à assurer sa place à l'intérieur même de l'institution intellectuelle. Toute personne attachée aux valeurs d'une pensée claire et rigoureuse ne pouvait qu'être étonnée du manque de sérieux de beaucoup d'affirmations présentées comme de nouvelles vérités.

L'idée du post-modernisme prend son origine dans la perception que l'on est entré dans une nouvelle phase de la société moderne. Jusque-là, celle-ci a été basée sur les idées venues des Lumières, au $18^{\text {ème }}$ siècle, avec la croyance en la Raison et l'Universalité. Mais les deux semblent avoir fait faillite. La Raison a conduit à la science et à la rationalisation de la société. L'Universalité a échoué, à l'égard du deuxième sexe, des colonies du tiers-monde, des personnes de couleur: il est clair que les personnes qui dirigent le monde sont des hommes blancs. Les idées d'universalité et de raison, de surcroît, ont conduit à la destruction, ou à la domination, d'autres types de culture. Un des aspects spécifiques des mouvements des années soixante et soixante-dix a été de vouloir faire reconnaître la légitimité de ces autres cultures: les femmes, les minorités, le tiers-monde.

Le courant post-moderne constitue, de fait, une galaxie sans homogénéité, dont je présente maintenant certains aspects importants.

\section{La déconstruction}

Cette notion a été proposée par le philosophe Jacques Derrida. Telle qu'elle est habituellement utilisée dans les zones de pensée post-moderne, c'est, de fait, surtout l'idée de remettre en question - autrement dit, d'être critique à l'égard de toute idée qui apparât comme évidente. Elle provient de cette nouvelle conscience - apportée par les sciences sociales - que nos idées sont implantées en nous par la société. 
L'adoption systématique d'une telle posture critique apporte un certain confort intellectuel: si je peux faire l'analyse de vos raisons, déconstruire vos valeurs, je gagne sur le plan intellectuel. Mais on peut aussi faire le choix de la responsabilité, et admettre que certaines de nos idées sont si fortes qu'on ne voudra pas les abandonner, en dépit de possibles explications psychiques ou sociales qu'on peut leur trouver.

Cela peut définir une gageure intellectuelle et morale plus intéressante que la première posture, qui a été si largement adoptée dans ces cercles qui se voulaient avant tout critiques. Elle signifie que l'on est prêt à reconnaître l'ambivalence habituelle de la société. Par exemple, on peut penser que la science tient une place trop importante dans la société et souffre de nombreux défauts. Mais il faut alors faire l'effort de distinguer dans la complexité de ce phénomène à la fois social et intellectuel ce qui doit être défendu - par exemple, sa façon à la fois intellectuelle et pratique d'aller vers la connaissance de la réalité - et ce qu'il faut condamner quelques-unes de ses applications aussi bien que certaines de ses déviations, par exemple une façon étroite d'être rationnel, qui refuse tout ce qui apparaît comme de l'irrationalité.

Ce n'est pas une surprise si la vague post-moderne a suivi la vague structuraliste et scientiste. Elle a consisté en une réaction contre un certain positivisme et son insistance à appliquer à la connaissance humaine et sociale les méthodes qui avaient réussi dans les sciences naturelles. Elle se complait à insister sur la fragilité de la logique et l'importance de la rhétorique: tout est vu à travers le prisme du «linguistic turn», c'est-à-dire comme une narration. L'herméneutique doit remplacer le positivisme.

L'idée de déconstruction a eu tellement de succès aux Etats-Unis que des bandes dessinées ont popularisé les idées de Derrida, Kristeva ou Lyotard.

\section{Les «Cultural studies»}

La critique de l'élitisme de l'Université a fait ressortir que la culture qui y était reconnue était principalement basée sur des œuvres produites par des hommes blancs. On retrouve ici l'influence d'une science sociale, à savoir l'ethnologie, qui a souligné le fait que toute population a une culture. Les Cultural studies ont commencé en Grande-Bretagne comme une réaction contre l'élitisme particulièrement poussé de ses universités. Elles voulaient étudier et revaloriser les cultures des minorités et des peuples opprimés. Le même mouvement a conduit, aux EtatsUnis, aux Black studies, puis aux Women's studies, puis aux Gay studies. Je pense personnellement que ce courant est tout à fait justifié. Cependant, certaines positions extrêmes vont jusqu'à refuser la qualité universelle de certains textes, et, en particulier, la validité spécifique de la science. Je discute ce point dans la prochaine section qui concerne plus particulièrement les études féministes.

\section{Le féminisme}

Le féminisme a connu un développement particulièrement fort aux Etats-Unis. Une question qui s'est posée est celle-ci: pourquoi si peu de femmes en science? Une première réponse consiste à pointer le machisme et la misogynie. Par exemple, la biologiste Barbara Mac Klintock, qui a reçu le prix Nobel seulement peu de temps avant sa mort, ou l'éminente mathématicienne Emma Noether, dont 
la qualité du travail avait été, dans les deux cas, reconnue pleinement par leurs pairs, n'ont pas eu droit à un poste dans l'Université. Les sociologues ont aussi montré l'importance des réseaux dans le processus de reconnaissance en science. C'est ainsi qu'une scientifique qui est mariée à un collègue a plus de chance pour sa carrière qu'une femme célibataire.

Mais les féministes radicales ont aussi renversé la question, et voulu regarder la science d'un point de vue féministe. Elles ont voulu aller jusqu'aux racines supposées «masculines» de la science - la volonté de dominer la nature, la dichotomie de la logique - alors que la féminité montrerait plus de subtilité et moins de besoin de domination... Etant une femme qui a pratiqué les mathématiques et la physique avec beaucoup de plaisir, et par ailleurs participé au mouvement de critique de la science des années soixante-dix ainsi qu'au mouvement de libération des femmes, je ne peux qu'être très sceptique devant ce qui m'apparaît comme la soumission à un radicalisme systématique ${ }^{12}$.

\section{La nouvelle sociologie et histoire des sciences}

Jusqu'à présent, l'histoire des sciences avait surtout été écrite par les scientifiques eux-mêmes, qui montraient le caractère cumulatif des découvertes de leur science. Le courant post-moderne a refusé ce type d'historiographie, qui était supposé ne donner la voix qu'aux vainqueurs. Une «position symétrique » a été proposée, qui insiste sur les controverses, les difficultés d'arriver à un consensus, l'usage d'arguments rhétoriques et d'autorité. Il s'agirait d'examiner d'un point de vue anthropologique, uniquement extérieur, comment les scientifiques «construisent» leurs données, les rendant ainsi sociales (et puis dites universelles). Une philosophie, le « constructivisme social», a ainsi remplacé la philosophie des Lumières basée sur les notions du vrai et du faux. Le succès de cette tendance tient de nouveau au fait qu'elle va à l'encontre de ce qui est perçu comme une domination de la science. Elle prétend dépasser le sens commun, et être ainsi, plus objective et sophistiquée.

C'est cette approche qui a mis en rage les scientifiques quand ils l'ont rencontrée. Leur pratique est de travailler dur et lentement pour approcher de la réalité des choses. Si leurs résultats ne sont que des constructions sociales, il n'y a plus de réalité. C'est alors que le biologiste Gross et le mathématicien Levitt lancèrent leur contre-attaque (cf. note 8).

\section{Les situations française / américaine}

Pourquoi les philosophes français ont-ils joué un tel rôle chez les postmodernes américains? et les «guerres de la science» ou l'»affaire Sokal» ont-elles été les mêmes dans les deux pays? De fait, la réponse à cette deuxième question est non. Les guerres de la science ont été avant tout américaines, et les attaques de Sokal et Bricmont ont été naturellement le plus fortement ressenties en France.

Voici une tentative de réponse à la première question. La place jouée par l'intellectualisme et les intellectuels est spécifique à la France. Leur influence est

12 J. Feldman, 1988, «Feminist critiques of science», Philosophy and Social Action, 14, 37-52. 1992, «Femmes, féminisme, science », L'année sociologique, 44, 425-440. 
importante. Il y a une tradition d'intellectuels s'engageant dans un combat pour différentes causes qu'ils estiment justes. Cela a été le cas célèbre du romancier Zola s'engageant pour Dreyfus. Après la deuxième guerre mondiale, les écrivains Simone de Beauvoir et Jean-Paul Sartre ont eu beaucoup de prestige, et ont rejoint le mouvement de mai et (pour de Beauvoir) le mouvement de libération des femmes. On peut aussi remarquer que trois de nos présidents de la $5^{\text {ème }}$ République ont une réputation réelle en tant qu'écrivains (de Gaulle, Pompidou, Mitterand).

Dans les années soixante-dix, deux figures intellectuelles avaient aussi un grand succès: des centaines de personnes se pressaient pour tenter de suivre leurs cours publics. Michel Foucault, Professeur au Collège de France (un titre parmi les plus prestigieux), était un philosophe qui s'est engagé dans un activisme au sujet des prisons. Jacques Lacan a popularisé une certaine sorte de psychanalyse, et, dans mon opinion, est le premier écrivain «post-moderne» célèbre. Il a délibérément opté pour un style peu clair, supposé rendre compte des subtilités de l'inconscient. Il avait l'habitude d'inclure dans ses développement de nombreux jeux de mots, une survivance de sa fréquentation du surréalisme. Son style très particulier a fasciné certains comme il en a irrité beaucoup d'autres.

Le statut reconnu des intellectuels en France va avec un système d'enseignement très compétitif, ce qui encourage une certaine tendance à l'élitisme et la brillance. La démocratisation de l'enseignement, qui va avec le développement des classes moyennes, a produit beaucoup de jeunes gens manquant de bases intellectuelles solides, qui peuvent être facilement séduits par des idées apparemment provocantes - ce qui signifie pour eux qu'ils sont dans le bon camp.

Si le phénomène a été intense en France dans les années soixante-dix, il semble qu'il ait été encore plus fort et prolongé aux Etats-Unis. Dans ce pays, il était nécessaire de rompre avec une philosophie positiviste à la domination pesante dans les sciences humaines. L'isolement des campus américains du reste de la société et l'absence d'une gauche politique intellectuelle ont encouragé les excès de la critique.

La mode du post-modernisme, du féminisme radical et du sociologisme réducteur des nouvelles études sur la science a peut-être aussi trouvé des limitations en France dans le fait que la recherche scientifique et le système d'enseignement y sont très largement publiques: les effets de mode sont sans doute moins forts que dans des systèmes où l'enseignement privé se doit de prendre en compte les desirata d'étudiants qui paient, et où les maisons d'édition tirent parti des engouements et aident à les propager.

\section{LES ASPECTS POLITIQUES ET ÉTHIQUES DE L'AFFAIRE SOKAL}

\section{L'engagement politique de Sokal}

Sokal a clairement dit que son canular avait un but politique. Il se présente comme de gauche et féministe. Il est donc d'accord sur le fait que plusieurs aspects de la science peuvent être critiqués. Mais la science est un phénomène social complexe, à la fois social et intellectuel, et le post-modernisme se fourvoie dans sa manière de l'attaquer. C'est un mauvais jeu intellectuel qui ne mène nulle 
part. Sokal aime à citer le linguiste Noam Chomsky dont les engagements politiques à l'extrême gauche sont bien connus. Tous deux estiment que la rigueur et la clarté de pensée sont des armes essentielles qui ne devraient pas être abandonnées aux classes dominantes. Il s'agit de ne pas confondre l'approche intellectuelle de la science et sa place dans la société; ni la rationalisation, phénomène politique, et la rationalité. Le refus de la rationalisation ne doit pas conduire au New-age. Le rejet des valeurs des Lumières va à l'encontre des intérêts des peuples.

\section{Ce qui a lui a été reproché}

Aux Etats-Unis, le reproche principal a été d'ordre politique. Sokal, en ridiculisant la revue Social Text, a divisé et affaibli la gauche universitaire, qui a déjà affaire à un contexte difficile.

En France, où la situation universitaire et politique n'est pas la même, comme montré plus haut, les reproches se sont placés surtout au plan intellectuel. Sokal et Bricmont menaient bataille contre la liberté de penser. En tant que physiciens, ils intervenaient dans des domaines philosophiques et des disciplines sociales dont ils ne connaissaient rien, et agissaient comme des censeurs, en refusant l'utilisation des métaphores, démontrant par là le caractère impérialiste des sciences exactes. Et pourquoi ne parlaient-ils pas des impostures de nombreux scientifiques? Mais il n'y eut pas de confrontation directe avec leurs critiques précises et détaillées.

Bien sur, ils firent savoir qu'ils n'étaient pas contre l'usage des métaphores. Mais, dans un style de pensée classique, un argument métaphorique aide à comprendre le plus difficile par le plus facile, le mieux connu. Si l'on fait l'inverse, en utilisant des images venues de disciplines très techniques et spécialisées, comme les mathématiques ou la physique théorique, cela ne peut être que pour de mauvaises raisons rhétoriques, en vue d'impressionner les gens avec l'apparence d'une érudition que l'on ne possède pas en réalité. Pour qu'une discussion réelle puisse avoir lieu, il vaut mieux utiliser des termes qui sont définis de façon aussi claires que possible.

Un autre reproche qui leur a été adressé a été qu'en utilisant l'arme d'un canular au lieu d'une argumentation, ils ont violé l'éthique scientifique habituelle. Pour leur défense, beaucoup de leurs partisans ont souligné l'absence d'effets de telles critiques, comparé à celui du canular. Le chimiste Pierre Laszlo a écrit: "Que faire lorsqu'une nouvelle scholastique discourt doctement de tout sujet, loin du réel, dans la ratiocination pure? Seul un énorme éclat de rire peut la déboulonner! $\gg^{13}$

\section{QUELQUES CONCLUSIONS}

\section{La difficulté de la pertinence idéologique}

La première leçon à tirer de ce débat est tout à fait générale: il s'agit de la difficulté bien connue d'atteindre le vrai et le juste. La critique de la place actuelle de

$13 \quad$ La Recherche, avril 1997 
la science dans la société est bienvenue, ainsi que celle de la domination et de l'oppression de cultures minoritaires. La réaction contre une domination intellectuelle et psychique est cependant malaisée. La plupart du temps, on a besoin d'un radicalisme pour se libérer des évidences de la domination. Mais tout se passe comme si l'effort de la libération entraînait trop loin. L'histoire, comme on sait, est remplie de révolutions qui, commençant sur des positions morales, n'ont pu éviter le sectarisme et d'autres violences.

C'est ce qui a pu être observé chez les courants critiques des années soixantedix: l'effort pour se libérer de l'idéologie dominante en a conduit beaucoup dans la séduction du radicalisme.

On a besoin de se sentir ensemble contre ceux qui nous dominent, et ceci conduit à un nouveau conformisme, ce qui est en contradiction avec une réelle liberté et la rigueur de pensée. Ceci peut être une premier pas inévitable en raison de la violence de la domination. La nouvelle étape devrait conduire à reconnaître que la réalité est le plus souvent bardée de complexités. Un esprit mûr psychiquement et intellectuellement accepte d'avoir à faire à ces complexités, mais, en revanche, il peut perdre beaucoup de son dynamisme ${ }^{14}$.

\section{Les deux cultures: de l'ignorance mutuelle au débat}

En 1959, un écrivain anglais, C.P.Snow, qui avait été physicien, a souligné l'existence de deux cultures, la scientifique et la littéraire ${ }^{15}$. Lui-même avait appartenu aux deux, pour la première, plutôt dans ses journées, et pour la seconde, dans ses soirées. Ces deux cultures s'ignoraient et se méprisaient mutuellement.

L'affaire Sokal a constitué une illustration récente et vivante de ces méconnaissances. En même temps, elle a introduit un nouvel élément, le désir de communiquer de l'une à l'autre. Les premiers contacts ont été plutôt rudes, mais Sokal et Bricmont se sont engagés délibérément dans de nombreuses discussions, montrant leur désir que l' «océan d'ignorance» entre les deux cultures dont parlait Snow commence à être comblé.

Le caractère multiculturel de la société moderne ne manque pas, de fait, de se refléter dans la cité académique, avec à la fois ses désirs et ses difficultés de communication et de compréhension mutuelle.

Certaines règles simples pour aider à cette communication sont valides dans les deux situations. On peut remarquer qu'un dialogue est d'autant plus fructueux que les deux parties ont le mieux conscience de leur identité personnelle, et qu'il aide en retour à mieux la définir. Dans le cas des sciences humaines et sociales, le problème est la faiblesse de leur identité, qui les a souvent conduites à vouloir imiter les sciences «dures» pour obtenir le statut rêvé de scientificité. En réaction contre ces imitations parfois serviles, le courant post-moderne a voulu «amollir» les sciences dures, pour tenter d'atteindre ainsi l'égalité.

Pour diminuer la possibilité des malentendus, il serait souhaitable qu'une base intellectuelle commune soit donnée aux étudiants. Tout étudiant devrait apprendre que, dans le champ de l'intelligence, plusieurs voies sont possibles, mais que chacune requiert du travail, de la rigueur, de l'attention. Il serait aussi essentiel de

14 On retombe ici sur l'adage bien connu: «Si jeunesse savait, si vieillesse pouvait».

15 C.P. Snow, 1959, 1963, The two cultures, Cambridge University Press. 
savoir dès le début que chacune de ces voies a ses propres limites et aussi ses dérives possibles. La science peut conduire au scientisme, la sociologie au sociologisme, etc. Chaque «isme» représente un type spécifique de dogmatisme, où une seule approche est considérée comme valable.

\section{A propos des valeurs universelles}

Le post-modernisme et l'affaire Sokal sont concernées par un problème politique et éthique qui va bien au-delà du monde académique. Il s'agit de l'idée d'universalité. En est-il ainsi qu'un mode de pensée qui recherche la clarté et la rigueur - avec la conscience de ses propres limites - est d'une valeur universelle? Personnellement, j'aurais tendance à le penser, mais mes contradicteurs peuvent faire valoir que j'ai été élevée dans une culture qui le valorisait. De la même façon, je pense que certaines des qualifications concernant le bien et le mal sont universelles. Je pense encore que beaucoup de résultats scientifiques, bien qu'ayant été acquis dans le cadre de civilisations spécifiques, sont en effet universels. Pour ne prendre qu'un exemple, choisi à dessein hors de la civilisation occidentale, tellement dominante actuellement: la découverte/construction du zéro a constitué un pas essentiel en arithmétique; faite par des savants indiens et arabes, elle est aujourd'hui utilisée par tous.

La rationalité est aussi, pour moi, une valeur universelle, qui contribue, jusqu'à un certain point, à une maîtrise de notre vie. Mais elle doit rester ouverte et ne pas nous couper d'autres modes de vie intellectuelle, tels la poésie ou l'art, par exemple. La rationalité est souvent pervertie, dans notre société, en rationalisation, qui n'est qu'instrumentale. Une trop forte rationalisation des soins médicaux, par exemple, conduit à oublier la spécificité du contact humain. Il en va de même dans les sciences humaines et sociales. Mais en dénonçant la rationalisation, on ne doit pas rejeter la raison, mais l'utiliser dans son combat.

3.

\section{L'AFFAIRE SOKAL (SUITE)}

Ce texte fait suite à «L'affaire Sokal »: un épisode de la méconnaissance entre cultures », L'année sociologique, 1999, 49, 245-270 ${ }^{16}$. On y examinait la méconnaissance entre les deux cultures - celle fondée sur les Lettres, la Philosophie, les Sciences sociales, d'une part et celle des chercheurs en sciences exactes de l'autre - à partir des réactions immédiates, dans la grande presse, à l'affaire Sokal, du nom du physicien à l'origine d'un canular qui a mis en difficulté une revue américaine "post-moderne», et co-auteur, avec un autre physicien, Jean Bricmont, d' un livre attaquant le laxisme de la pensée de certains intellectuels célèbres: Impostures intellectuelles. 1997, Paris, Editions Odile Jacob; 2ème édition, Livre de Poche, 1999.

16 Il devait paraître en 2000 dans le numéro 1, vol. 50, de L'Année sociologique, mais a été supprimé au dernier moment, faute de place. 
Cette «suite» examine les premières réactions plus réfléchies parues dans le livre collectif de Baudoin Jurdant (le plus intéressant dans l'optique qui est la mienne), un livre d'Yves Jeanneret et des articles d'un numéro des Temps Modernes consacré à l' "affaire».

Bien d'autres livres, articles et débats ont eu lieu après l'écriture de ce texte. $J$ 'en cite quelques-uns en note ${ }^{17}$.

Sous la direction de Baudouin Jurdant: Impostures scientifiques; Les malentendus de l'affaire Sokal.

Paris, La découverte /Alliage, 1998, 332p. 16 contributeurs.

Yves Jeanneret: L'affaire Sokal ou la querelle des impostures PUF, 1998, 274 p.

Les Temps Modernes: Retour sur l'affaire Sokal. Le vrai débat

Juillet-Août-Septembre 1998, n 600, 3 contributeurs

L'automne nous a apporté une nouvelle floraison concernée à l'affaire Sokal, livres et articles, articles en livre ${ }^{18}$. Il s'agirait, cette fois - sauf pour Yves Jeanneret dont le travail se veut avant tout descriptif - de se distancier des polémiques «médiatiques», de creuser les problèmes, de cerner les enjeux, de dépasser les malentendus. Bref, comme l'annonce la revue Les Temps Modernes, d'engager le «vrai débat». Ce projet est, bien entendu, le bienvenu, même si la plupart du temps, il nous semble encore rester en chemin.

L'ensemble nous apporte toutefois quelques éclairages importants, en particulier sur le contexte de la querelle et les courants qui ont fait l'objet des attaques de Sokal.

Le livre collectif publié sous la direction de Beaudoin Jurdant se présente explicitement comme «la réponse de la bergère au berger». Il commence donc par une attaque en règle contre les défaillances des scientifiques.

Pour le physicien théoricien Jean-Marc Lévy-Leblond qui, sans surprise, ouvre l'attaque, si «on ne saurait laisser sans réponse les mésusages caractérisés des concepts scientifiques, cette critique cependant doit commencer par être autocritique». Pour cet auteur, ce serait souvent les physiciens eux-mêmes dont «la médiocrité de la pratique langagière » met en circulation des notions qui donnent prise aux malentendus: ainsi, le célèbre «principe d'incertitude » de la Mécanique Quantique, qui correspond en fait à des «inégalités » découvertes par Heisenberg; ou encore la «relativité » d'Einstein qui, dans «sa conception moderne, met l'accent sur les invariants (absolus) de l'espace-temps». Les fameux big-bang, trous

17 Comme il n'est pas question d'exhaustivité, je me borne à citer deux ouvrages, l'un écrit, l'autre dirigé, par deux philosophes qui ont la particularité d'avoir pris fait et cause pour Sokal: Jacques Bouveresse, Prodiges et vertiges de l'analogie : de l'abus des belles lettres dans la pensée, Paris, Raisons d'agir, 1999. - Angèle Kremer-Marietti (dir.): Ethique et Epistémologie autour du livre Impostures Intellectuelles de Sokal et Bricmont, Paris, L'Harmattan, 2001. - Je signale aussi un autre article que j'ai écrit sur la question: «Les savoirs aujourd'hui: pour un travail d'explicitation». In : Angèle Kremer-Marietti, op. cit. 39-64.

18 Je ne ferai que signaler ici, sans le discuter, le livre de Marc Richelle professeur de psychologie expérimentale à l' Université Catholique de Louvain: Défense des sciences humaines. Vers une désokalisation? Mardaga, 1998. Sa «défense» ouvrirait le débat directement sur les problèmes de scientificité des sciences de l'homme, alors que l'affaire Sokal, sur laquelle je me concentre ici, met principalement en ligne les sciences «dures » d'un côté et certains courants de pensée dits postmodernes. 
noirs, particules charmées, chaos, et autres catastrophes voient leur «réel intérêt intellectuel... masqué et déformé plutôt que révélé et éclairé par ces appellations non contrôlées ». L'auteur évoque, en contrepartie, les «prédécesseurs du XIXe... grands créateurs de mots...alliant raison et imagination...(avec) une explicite description critique ».

Il est aussi facile de pointer certaines «tentations de syncrétisme théologicoscientifique moderne ${ }^{19}$. Mais surtout de dénoncer l'arrogance d'une discipline qui se prend - et, il faut le souligner, est prise par bien d'autres encore que ses propres praticiens - pour le parangon de la Science.

L'ancienne «vulgaire chimiste» (aux yeux des physiciens) qu'a été Isabelle Stengers soulève à ce propos un lièvre important. Il faut cesser, dit-elle, d'identifier «réalité» et «réalité physique». Car, «dès lors qu'il parle des «lois fondamentales de la nature», n'est-ce pas le physicien lui-même qui déclare la guerre, et la déclare à tous?». Lévy-Leblond dénonce les illusions d'un Weinberg à découvrir «l'ultime théorie », celle d'un Hawking, «l'un des maîtres de la cosmologie moderne» qui ajoute « un fantasme d'omnipotence à celui d'omniscience», ou, plus généralement, les prétentions de la science à pouvoir répondre à tous les problèmes.

Sokal se place dans cette lignée quand, avec pas mal de candeur, il défend une «vue scientifique» du monde ${ }^{20}$, basée sur la logique et la confrontation au réel. On peut ici lui reprocher d'ignorer l'expérience marxiste et communiste ou, d'une manière plus générale, comme le fait Georges Guille-Escuret dans son article des Temps Modernes, qui concerne «les sciences humaines en tenaille», «son indifférence flagrante aux sciences humaines ».

Attaque encore contre «l'autorité de la science», un thème justifié, mais malheureusement développé ici dans une contribution qui ne fait qu'illustrer certaines confusions que l'on reproche aux post-modernes: car si la résistance, de la part de l'«establishment» mathématique de l'époque, à l'invention d'espaces non-euclidiens est un épisode de l'histoire des mathématiques tout à fait passionnant, le fait que cette résistance ait été due à l'influence des idées du philosophe Kant viendrait plutôt apporter de l'eau au moulin des physiciens qui, comme Weinberg ou Feynman, se dressent contre la philosophie.

On passera sur l'analogie naïve faite avec les attaques actuelles subies par les social science studies - tout ce qui est rejeté par l'orthodoxie scientifique n'étant pas pour autant génial ou vrai. Est également faussée la démonstration de «l'inéluctable historicité de la constante $\pi$, qui intervenait dans la parodie de Sokal et que veut ici défendre le même auteur, confondant celle-ci avec les variations possibles de la formule de la circonférence d'un cercle selon le type d'espace utilisé.

Bien évidemment, le fait que $\pi$ soit une constante ne signifie pas qu'elle n'ait pas d'histoire, ni le fait qu'elle ait une histoire ne signifie pas qu'elle ne soit pas une constante. Le mathématicien calculera avec la constante, l'historien des sciences s'intéressera à son histoire, il n'y a rien ici que de très simple. Qu'un mal-

\footnotetext{
19 On consultera à ce propos le très beau livre de Gilles Gaston Granger L' 'irrationnel, Paris, Editions Odile Jacob, 1998.

20 Alan Sokal: A plea for Reason, Evidence and Logic, New Politics, 6(2), pp. 126-129.
} 
entendu pareil puisse avoir lieu, mis en place par un courant qui se targue par ailleurs d'insister sur les «points de vue», témoigne de la confusion où les «subtilités» de certaines «études sur la science» ont pu aboutir, à la suite de leur volonté de se distinguer de leurs prédécesseurs en prenant en compte les «contenus » mêmes de la science, ainsi que l'indique sans ambiguïté Michel Callon. La «défense et illustration des recherches sur la science» qu'il nous présente semblerait destinée aux instances institutionnelles dispensatrices de crédits et de reconnaissance officielle. S'il y reconnaît qu'un certain sociologisme outrancier a parfois sévi, il n'en demeure pas moins fidèle à la fameuse notion de «symétrie », refusant ce qui fait la spécificité de la science. Désireux d'éviter l'opposition entre réalisme et relativisme, il est obligé d'introduire les notions complexes de «chaînes de traduction». Et l'on est soulagé lorsque Isabelle Stengers, qui se place résolument dans le camp des «constructivistes sociaux » et «penseurs postmodernes » attaqués par Sokal, mais dont le très beau texte entend aller vers la paix, explique longuement à ses amis, en se plaçant sous l'égide de Stephen J. Gould, sympathisant constructiviste par ailleurs, la spécificité des controverses scientifiques: il s'agit de la possibilité d'une solution que «cette référence réputée infâme», autrement dit la «réalité», mise ici entre guillemets, prudence de philosophe oblige, permet d'obtenir. Il est alors possible de dépasser «l'état de confusion intellectuelle totale» où nous laisse la description purement constructiviste d'une trouvaille scientifique, dont les «vaincus peuvent se réjouir autant que les vainqueurs» et «célébrer» ensemble les «triomphes de la connaissance humaine», qui permettront d'aborder de nouvelles questions. Gould utilise pour cela le terme de «douce victoire», que Stengers reprend à son compte.

«Célébration», c'est également le terme que reprend Jean-Michel Salanskis: «il me semble exigible de toute réflexion sur la science qu'elle soit, entre autre choses, une célébration de la pensée scientifique comme telle... l'école pourvoyeuse de méthode, de vérité et de conceptualisation inouïes qu'est la science».

Pour Stengers, le refus des sociologues de la science d'admettre les valeurs que ce type de travail représente pour les scientifiques constitue une «conduite grossière et insultante». Et de même que les géologues, dans l'exemple abordé à travers Gould, se préoccupent des réponses de la Terre, les sociologues devraient se préoccuper de la réponse de leurs «objets d'études».

«Encore un effort», demande le phycisien David Mermin à ses collègues, les engageant à tenter de comprendre ce que veulent montrer les nouveaux sociologues de la science. «Encore un effort», ajouterai-je en direction de ces derniers, pour reconnaître la véritable passion pour la réalité qui anime aussi bien le naturaliste cherchant à mieux connaître les crotales, l'archéologue découvrant avec soin quelques morceaux de poterie qui lui permettront de reconstituer le puzzle du passé, ou encore le physicien des hautes énergies traquant avec ses accélérateurs une réalité devenue étrangère au sens commun, certes, mais tenue ensemble par un réseau ultra-serré de cohérences.

Le reproche principal qui s'exerce contre Sokal et Bricmont est de ne rien comprendre à la subtilité de la pensée philosophique ou à celle des sciences humaines (que l'on ne distinguera pas, en règle générale). «C'est... contre cet éléphant discursif et maladroit qu'il faut défendre la pensée...» écrit Jurdant dans son 
introduction. Juliette Simont, dans les Temps Modernes, intitule son article «La haine de la philosophie »: "Qu'il y ait de bons et de mauvais philosophes, c'est certain. Mais jamais la simple présence dans leur discours d'une formule scientifique mal comprise ne fera la décision ».

Défense de la philosophie, qui entretiendrait avec la vérité et l'erreur des rapports totalement différents de ceux des sciences exactes, avec, selon Deleuze, la disparition d'une opposition totale entre ces termes.

Défense des auteurs nommément attaqués, dont aucun par ailleurs ne participe à ces textes, mais qui y trouvent des alliés décidés: Lyotard, Derrida, Lacan, Debray «godelisant» (alors que lui-même a pris des distances par rapport à cette époque), Latour commentant la relativité d'Einstein...Ou bien leurs mathématiques feraient effectivement sens dans leur démarche, ou bien elles seraient une sorte d'aide lors du cheminement difficile de la pensée. Pas la moindre acceptation du reproche qui leur a été fait d'utiliser les mathématiques pour impressionner un public crédule.

Ce sont donc, dans ces domaines différents de la science, d'autres règles de jeu qui auraient cours. Mais y a-t-il véritablement règles? c'est la (bonne) question que pose Salanskis.

Celui-ci admet, par exemple, que dans les sciences cognitives, qui se situent au carrefour de trois disciplines constituées, la linguistique, la philosophie et les neurosciences, «nous sommes sans règles », et qu'aucune véritable interdisciplinarité ne s'est construite. Reste alors la recommandation de «garder l'orthodoxie rationnelle sans pouvoir se référer à des tables strictes ».

Une règle est pourtant proposée, et même une «norme»: «la norme de l'herméneutique ». Elle consiste à tenter de comprendre ce que dit l'autre, et représente une déontologie de la lecture, mais aussi de la critique. Il s'agit de refuser, par exemple, le «mauvais ricanement du mathématicien à propos du physicien» qui utilise constamment des mathématiques pas aussi «pures» que le premier le voudrait, (ici est donné l'exemple bien connu de Dirac) ainsi que «la violente jubilation qu'on a de son habitus et la supériorité qu'on lui prête sur tous les autres ».

Ces règles de respect mutuel rejoignent l'insistance de l'éthique actuelle sur le respect de l'autre même si, comme le souligne Stengers, l'environnement universitaire est loin de les encourager. Mais Salanskis s'aventure beaucoup plus loin: il entend faire reconnaître une «irréductibilité de la compétence rhétorique » qui ne devrait pas plus être bridée que celle du surdoué en mathématiques, «au nom de la même morale de la protection de l'excellence ».

Ici sont posés quelques questions importantes et nouvelles qui, sans nul doute, découlent à la fois du contexte social global que de l'accroissement de la Cité intellectuelle, où le contrôle qu'exerçaient jadis les «mandarins » est affaibli, tandis que des groupes peuvent se constituer et se faire légitimer en jouant les règles apparentes de la science.

Peut-être faut-il évoquer ici une sorte de «libéralisme» de la pensée, avec les droits accordés à celui qui peut le mieux séduire. Nul souci pédagogique qui, il faut le rappeler, anime profondément l'entreprise de Sokal. Cette absence semblerait bien aller de pair avec la distinction apportée par plusieurs des auteurs, et tout particulièrement par Stengers, portant sur l'existence de deux «classes intellectuelles »: les «humbles travailleurs de la preuve»d'un côté, et les «élites» à la pensée «sophistiquée, subtile, cultivée...ironique» de l'autre. Aux premiers, les 
exigences d'une morale austère du travail intellectuel - on ne peut faire n'importe quoi - et, soulignerai-je de nouveau, un authentique désir pédagogique, avec la défense d'un «bon sens » que récusent avec dédain les esprits se prétendant plus raffinés. Les premiers s'en prendraient ainsi à ceux qui leur apparaissent comme des «parasites irresponsables jouissant sans travail du luxe des mots». Etrange retournement d'une situation où l'on avait d'abord vu apparaître une science arrogante et aux prétentions hégémoniques.

Il se pourrait que ce «libéralisme» d'un courant qui revendique si fortement le droit à une pensée sans contraintes aille effectivement de pair avec le libéralisme victorieux de notre société actuelle. A moins qu'il ne s'agisse - les deux n'étant peut-être pas incompatibles - du «libertarisme » issu des années 68. Alors s'élève la révolte contre le scientisme des années d'après-guerre, comme le rappellent Amy Dahan-Dalmedico et Dominique Pestre dans leur contribution. Pour Patrick Petitjean, qui nous décrit les mouvements de critique des sciences, Sokal et Bricmont se situent dans la lignée de la gauche scientiste des années trente, qui a du mal à faire le deuil de la séparation actuelle entre science, progrès et socialisme.

L'article qui clôt le livre évoque une autre affaire, l'«affaire Jean Clair». Ce sont ici les critères de l'appréciation esthétique qui apparaissent en crise. Ce rapprochement laisserait entendre que l'on a bien affaire aux retombées d'une évolution de la société globale.

Plusieurs auteurs nous offrent une vue plus approfondie des mouvements dits postmodernes des pays anglo-saxons.

L'anthropologue Bernard Traimond nous explique comment «au lieu de maintenir la fiction qu'au sein de l'institution, seule la qualité des arguments l'emporte, les anglo-saxons ont préféré développer une nouvelle discipline »: son objet porte sur les cultures contemporaines, en réaction avec la conception élitiste de la culture qui prédominait chez les britanniques chez qui elles sont nées; sa démarche repose sur le refus du positivisme, et son centre d'intérêt privilégié concerne l'étude des formes de pouvoir.

Mais «récentes, vulnérables et hétérogènes», leur éclectisme leur font courir des risques d'appauvrissement, et elles présentent trop souvent un «bric-à-bric sans maîtrise ».

De plus, pour Michel Pierssens, professeur à Montréal, le passage outre-atlantique les a mises dans le moule du «star system universitaire américain », qui permet d'obtenir les postes les plus prestigieux dans les meilleures universités avec les salaires les plus élevés et les à-côtés les plus confortables grâce à la surenchère dans la dénonciation de ces mêmes avantages ».

Dans ces mouvements qui se veulent contestataires, la notion de social construction devient «un poncif méthodologique dégradé en simple symbole d'allégeance au mouvement », l'évocation de Derrida un «signe de ralliement » et Latour, «une figure élevée au rang d'autorité dogmatique».

Stengers elle-même recommande à ses amis de ne plus réagir «comme un chien de Pavlov au bruit d'une sonnette» à certains mots, tel que le pouvoir, mais on pourrait y ajouter les mots de «réalité », d' «universalité» et d'«objectivité », tous tenants de cette vision classique de la science que veut mettre à mal le courant en question. 
Yves Jeanneret, dans son livre, nous propose également quelques informations sur le postmodernisme américain avec, en particulier, la reproduction de quelques pages de «comics», petits guides illustrés, telles une Introduction au postmodernisme ou un Derrida pour débutants, où l'on voit apparaître dessinés Kristeva, Derrida et Lyotard.

Une autre question importante abordée concerne la «mise en culture » de la science, comme aime à l'exprimer Lévy-Leblond. Trouvant dommageable la coupure existant entre la science et le reste de la société, il est prêt à accepter le «risque permanent du malentendu ». De la même façon, Jurdant, dans son introduction, considère que la polysémie qui affecte les notions scientifiques, dès lors qu'elles sortent de l'environnement où elles ont été créées, participe à la culture, et «restaure la texture imaginaire de la pensée scientifique elle-même».

Mais n'est-ce pas payer parfois un peu cher, au plan de la qualité intellectuelle, cet «élargissement du cercle», selon l'expression d'Andrée Bergeron, géophysicienne? et ne s'agirait-il pas en définitive d'une sorte de condescendance proche de ce mépris tant reproché à Sokal, que d'estimer que les non-scientifiques sont incapables de comprendre, sinon les divers éléments de la science - ce dont les scientifiques sont eux-même incapables, en raison de la spécialisation à outrance où celle-ci est parvenue - mais ce qui fait sa spécificité propre où, si l'imagination joue un rôle, c'est pour être aussitôt contrôlée dans cette obsession de coller à la réalité? Il y aurait là comme une démission du souci de «vulgarisation», une entreprise certes pleine d'embûches, mais qui animait passionnément notre dixneuvième siècle si scientiste.

Yves Jeanneret, professeur de sciences de l'information et de la communication, s'intéresse justement à «la façon dont les divers savoirs circulent, se légitiment, se rencontrent ou s'évitent». C'est ce qu'il nomme la «dimension triviale de la culture», dans un sens évidemment non péjoratif. Il a donc examiné près de trois cents textes intervenant sur l' "affaire» parus dans les medias ou sur des sites Internet. Mais ce mode d'approche qui privilégie l'extensif au détriment d'une réflexion plus approfondie donne trop souvent une impression de confusion, et d'un aplatissement des problèmes en question. On peut, là encore, être séduit ou pas par le brillant de l'auteur et son goût des formules. «Invocation du fait, triomphe du texte », la forme du titre de la première partie est reprise ensuite avec les oppositions suivantes: réel/fiction, constante/ variable, science/ littérature, lumière/ spectre. On notera, parmi les nombreux éléments de discussion effleurés, outre l'examen du postmodernisme américain déjà mentionné, le dénombrement de notions pas toujours bien choisies à mon avis, et celui des noms de personnes, où l'on s'aperçoit que Lacan détiend toujours la vedette devant Derrida, Latour et Deleuze.

L'affaire Sokal, commencée aux USA comme un jeu de duperie, continuée en France avec une accusation grave, celle d'imposture, a conduit à une violence d'expression rarement atteinte dans les affrontements intellectuels autrement plus feutrés en général. Ainsi en témoigne l'échantillon suivant des termes utilisés: mépris, haine, horreur, rire, ricanement, exaspération, offense, désinvolture, arrogance, mauvaise foi, indignation, injure, effarement, infâme, ignoble...

Comme le signale Jurdant dans son introduction, la «remise en cause d'équilibres instables» (à savoir l'ignorance réciproque entre scientifiques d'une part, 
philosophes et praticiens des sciences de l'homme de l'autre) a provoqué des blessures qui concernent plus les «identités» elles-mêmes que les idées.

On sait gré à certains auteurs d'avoir su faire état de leurs propres blessures et de ne pas seulement avoir joué la contre-attaque: blessure de la scientifique lisant un roman qu'elle apprécie jusqu'au moment où celui-ci se plait à décrire «avec une grande précision un tremblement de terre et ses séismes». Andrée Bergeron a alors une réaction «épidermique» de spécialiste, s'irritant « de ce qui lui semble inexact, pas juste, fantaisie d'auteur».

Ecriture également personnelle que celle de Salanskis, qui a fait des études de mathématiques, qui pourrait trouver à s'identifier à Sokal, mais qui, devenu philosophe, et travaillant dans les sciences cognitives, s'est senti «blessé, touché, bouleversé, gêné »; et reconnaissant, à la fin de son article, qu'il n'y a pas suivi luimême les règles de déontologie de la lecture et de la critique qu'il y a introduites. Cette personnalisation, à côté des discours intellectuels, contribue, autant qu'à l'humanisation du débat, à une meilleure compréhension de l'intensité vécue des choix intellectuels en cause dans la polémique.

Post-Scriptum: Les Cahiers Rationalistes, dans leurs numéros d'avril à décembre 1998, font paraître, à la suite d'un colloque pro-sokalien, sept articles, parmi lesquels des réactions de Sokal et Bricmont à la polémique. Signalons plus particulièrement l'insistance du physicien Evry Schatzman sur la différence entre les sciences de la nature et les sciences de l'homme et l'évocation par le physicien J.C.Pecker de certains éléments de l'histoire de la pensée «anti-science». Jacques Bouveresse, dans un long article ( $n^{\circ} 528$ et 529), présente la réelle signification du théorème de Gödel sur l'incomplétude, parle de la «morgue littéraliste», néologisme construit à partir de «scientiste», et fustige le manque de tradition, en France, d'une culture du débat argumenté.

GEMAS-CNRS

Paris 